\title{
Accretion rates in Herbig Ae stars ${ }^{\star}$
}

\author{
R. Garcia Lopez ${ }^{1,2}$, A. Natta ${ }^{1}$, L. Testi ${ }^{1}$, and E. Habart ${ }^{1,3}$ \\ 1 INAF - Osservatorio Astrofisico di Arcetri, Largo Fermi 5, 50125 Firenze, Italy \\ e-mail: natta@arcetri.astro.it \\ 2 Università degli Studi di Roma "Tor Vergata" - Dipartimento di Fisica, via della Ricerca Scientifica 1, 00133 Roma, Italy \\ 3 Université Paris-Sud, 91405 Orsay, France
}

Received 9 May 2006 / Accepted 29 August 2006

\section{ABSTRACT}

\begin{abstract}
Aims. Accretion rates from disks around pre-main sequence stars are of importance for our understanding of planetary formation and disk evolution. We provide in this paper estimates of the mass accretion rates in the disks around a large sample of Herbig Ae stars. Methods. We obtained medium resolution $2 \mu \mathrm{m}$ spectra and used the results to compute values of $\dot{M}_{\text {acc }}$ from the measured luminosity of the $\mathrm{Br}_{\gamma}$ emission line, using a well established correlation between $L\left(\mathrm{Br}_{\gamma}\right)$ and the accretion luminosity $L_{\text {acc }}$.

Results. We find that $80 \%$ of the stars, all of which have evidence of an associated circumstellar disk, are accreting matter, with rates $3 \times 10^{-9} \lesssim \dot{M}_{\text {acc }} \lesssim 10^{-6} M_{\odot} / \mathrm{yr}$; for 7 objects, 6 of which are located on the ZAMS in the HR diagram, we do not detect any line emission. Few HAe stars $(25 \%)$ have $\dot{M}_{\text {acc }}>10^{-7} M_{\odot} /$ yr.

Conclusions. In most HAe stars the accretion rate is sufficiently low that the gas in the inner disk, inside the dust evaporation radius, is optically thin and does not prevent the formation of a puffed-up rim, where dust is directly exposed to the stellar radiation. When compared to the $\dot{M}_{\text {acc }}$ values found for lower-mass stars in the star forming regions Taurus and Ophiuchus, HAe stars have on average higher accretion rates than solar-mass stars; however, there is a lack of very strong accretors among them, probably due to the fact that they are on average older.
\end{abstract}

Key words. stars: formation - stars: circumstellar matter - stars: pre-main sequence - accretion, accretion disks

\section{Introduction}

Pre-main sequence stars accrete matter from their circumstellar disks for a large fraction of their life, until the disk is accreted entirely onto the star or is dissipated by a number of potentially competing processes, such as dynamical perturbations by close companions, photoevaporation by the host or nearby stars, or planet formation. Although the amount of matter accreted is small compared to the mass of the central object, accretion of matter through the disk has important consequences on the physics of the disk itself and on the properties of the planetary systems that the disk may form.

The mass accretion rate through the disk is reasonably well known for pre-main sequence stars with masses from about one solar mass (T Tauri stars or TTS), down to brown dwarfs (BDs). Results can be found, e.g., in Gullbring et al. (1998), Muzerolle et al. (1998, 2003, 2005), White \& Ghez (2001), Natta et al. (2004, 2006), Mohanty et al. (2005).

It has been much more difficult to determine $\dot{M}_{\text {acc }}$ in stars more massive than about $1 M_{\odot}$. Recently Calvet et al. (2004) obtained the accretion rate in nine young pre-main sequence stars with masses in the interval 1.5-3.7 $M_{\odot}$ (intermediate mass TTS or IMTTS) using HST and optical spectra. They found an average accretion rate of $\sim 3 \times 10^{-8} M_{\odot} / \mathrm{yr}$, with a spread of an order of magnitude at least. Muzerolle et al. (2004) derived a similar

* Based on observations collected at the Paranal Observatory, European Southern Observatory, Chile, as part of ESO programme 073.C-0184. value of $\dot{M}_{\text {acc }}$ for the $\sim 2 M_{\odot}$ star UX Ori by fitting its $\mathrm{H}_{\alpha}$ profile with magnetospheric accretion models.

We report in this paper measurements of the accretion rate in a large sample of Herbig AeBe objects (intermediate mass pre-main sequence stars), mostly of spectral type A (HAe). We derive the accretion rate from the luminosity of the $\mathrm{Br}_{\gamma}$ line seen in emission in pre-main sequence accreting objects. Calvet et al. (2004) have shown that the correlation between the hydrogen IR line luminosity and the accretion luminosity found in TTS and BDs (Muzerolle et al. 1998; Natta et al. 2004) extends to their sample of IMTTS (see also van den Ancker 2004). Establishing the luminosity of the hydrogen near-IR recombination lines as proxy of the accretion luminosity has been extremely important, since it has made possible to derive accretion rates with reasonably good accuracy in objects where measuring the UV veiling is challenging. This is the case, for example, of very young star-forming regions, such as Ophiuchus, where the high extinction is the limiting factor (e.g., Natta et al. 2006), but also of the Herbig AeBe stars, where it is difficult to separate the UV excess emission from the hot photospheric continuum.

The main purpose of this work is to estimate $\dot{M}_{\text {acc }}$ in objects with disks for which we are currently obtaining near-IR interferometric data with the ESO VLTI (AMBER GTO ${ }^{1}$; see also Malbet et al. 2006; Tatulli et al. 2006). Knowledge of $\dot{M}_{\text {acc }}$ is crucial for understanding the disk geometry, in particular the nature of the disk inner rim (e.g., Natta et al. 2001; Dullemond et al. 2001; Isella \& Natta 2005), which can only form if the

\footnotetext{
${ }^{1}$ http://www.eso.org/observing/proposals/gto/amber/
} 
accretion rate is lower than a critical value of the order of $10^{-7} M_{\odot} / \mathrm{yr}$ (Muzerolle et al. 2004). For low $\dot{M}_{\text {acc }}$, the inner gaseous disk is optically thin and does not prevent the direct stellar irradiation of the inner dust edge of the disk, thus giving raise to the puffed-up rim. Secondly, we are interested in comparing the results to the accretion properties of the lower-mass objects studied in Taurus and Ophiuchus. In both regions it has been found that the mass accretion rate $\dot{M}_{\text {acc }}$ is a strong function of the mass of the central object, increasing roughly as $M_{\star}^{1.8-2.0}$ (e.g., Natta et al. 2006, and references therein; but see also Clarke \& Pringle 2006). The causes of this behaviour are not understood, and are matter of debate (Muzerolle et al. 2005; Natta et al. 2006; Alexander \& Armitage 2006; Dullemond et al. 2006). The HAe stars are scattered in different regions of the sky and are, on average, older than objects in Ophiuchus and Taurus; they may provide interesting information on the dependence of the disk accretion on age and environment.

The outline of this paper is as follows. The observations and data reduction are described in Sect. 2; method and results are presented in Sect. 3 and discussed in Sect. 4. Conclusions follow in Sect. 5.

\section{Observations and data reduction}

Medium resolution $(R \sim 9000)$ spectra of all stars in our sample were obtained with the ISAAC spectrograph at the Antu $8.2 \mathrm{~m}$ VLT unit telescope in service mode in spring 2004. The spectral region covered by our spectra included the $\mathrm{Br} \gamma$ and the $\mathrm{H}_{2}(1-0) \mathrm{S}(1)$ lines, which was not detected in any of the objects of the sample. All spectra were obtained in moderately poor conditions (seeing $\sim 1.5$ arcsec and non photometric transparency), as these were adequate for the scope of the project. As a consequence, we did not attempt to obtain an accurate flux calibration of the spectra.

The raw data were wavelength calibrated using daytime arcs and rectified using data from the ISAAC calibration plan. After aligning and coadding the dithered integrations on each target, the spectra were extracted and telluric absorption and instrumental throughput were corrected using observations of telluric standards obtained by the observatory staff during the same nights as the science observations. The details of the ISAAC calibration plan, the reduction process and the instrument characteristics can be found on the ESO web pages ${ }^{2}$.

The portion of the spectra around $\mathrm{Br}_{\gamma}$ are shown and briefly discussed in the appendix. In each spectrum we measured the equivalent width of the $\mathrm{Br} \gamma$ line; the measurements are given in Tables 1 and 4, together with the $1 \sigma$ uncertainties.

Tables 1 and 4 report also the star name and properties. Spectral type and distance are compiled from the literature (e.g., van den Ancker et al. 1997, 1998; Testi et al. 1998; Meeus et al. 2001; Rodgers 2001; Hernandez et al. 2005, and references therein). Extinction and luminosity are computed from the $V,(B-V)$ magnitudes as described in Testi et al. (1998); stellar masses are derived by comparing the location of each star on the HR diagram to the evolutionary tracks of Palla \& Stahler (1993).

\section{Method and results}

We derive the accretion luminosity $L_{\mathrm{acc}}$ of each star from the luminosity of $\mathrm{Br}_{\gamma}$, following the relation derived by

\footnotetext{
${ }^{2}$ http://www.eso.org/instruments/isaac
}

Calvet et al. (2004) for a sample of objects ranging in mass from about $4 M_{\odot}$ to BDs:

$\log L_{\mathrm{acc}} / L_{\odot}=0.9 \times\left(\log \left(L\left(\mathrm{Br}_{\gamma}\right) / L_{\odot}\right)+4\right)-0.7$

where $L\left(\mathrm{Br}_{\gamma}\right)$ is the luminosity of the $\mathrm{Br}_{\gamma}$ line emitted by the circumstellar gas. Note that Eq. (1) is an empirical relation, where $L_{\text {acc }}$ is measured from the observed veiling, i.e., independently of any assumption on the origin of the $\mathrm{Br}_{\gamma}$ emission. It has been derived from objects with $L\left(\mathrm{Br}_{\gamma}\right)$ between $\sim 3 \times 10^{-6}$ and $\sim 3 \times 10^{-3} L_{\odot}$; the scatter of $L_{\text {acc }}$ for any given $L\left(\mathrm{Br}_{\gamma}\right)$ is typically a factor \pm 3 (see also van den Ancker 2004).

In order to compute $L\left(\mathrm{Br}_{\gamma}\right)$, we need to extract the equivalent width of the circumstellar component of the line from the observed one. In HAe stars, the observed flux is the sum of the stellar photospheric emission, which for A stars shows broad and rather strong absorption in the hydrogen lines, of the emission of the circumstellar gas and of the emission of the disk. Following Rodgers (2001), we assume that the star dominates the observed $V$ emission. The equivalent width of the photospheric component, corrected for the $K$-band veiling, is computed for each object with spectral type earlier than A8 using the template spectra of Rodgers (2001). For objects of later spectral type, for which we did not have spectra of template stars of suitable resolution, we compute $W\left(\mathrm{Br}_{\gamma}\right)_{\text {circ }}$ from the expression:

$W\left(\mathrm{Br}_{\gamma}\right)_{\text {circ }}=W\left(\mathrm{Br}_{\gamma}\right)_{\mathrm{obs}}-W\left(\mathrm{Br}_{\gamma}\right)_{\text {phot }} 10^{-0.4 \Delta K}$

where $W\left(\mathrm{Br}_{\gamma}\right)_{\text {obs }}$ is the observed equivalent width, $W\left(\mathrm{Br}_{\gamma}\right)_{\text {phot }}$ the equivalent width of the photospheric $\mathrm{Br}_{\gamma}$ line of a template with the same spectral type (see Table 3; values from Rodgers 2001 and Wallace \& Hinkle 1997) and $\Delta K$ the disk continuum emission, computed subtracting from the observed $K$ magnitude the photospheric one. All the relevant parameters used in the derivation are given in Table $1 . K$ magnitudes are from $2 \mathrm{MASS}^{3}$; note that none of the quantities used in Eq. (2) has been measured simultaneously.

Of the 32 targets, 4 are very luminous objects of spectral type early B. Three of them have $\mathrm{Br}_{\gamma}$ in emission, one in absorption. Table 4 refers in Col. 3 a lower limit to the line luminosity, derived from the observed $\mathrm{Br}_{\gamma}$ equivalent width, i.e., without correcting for the photospheric absorption. For the three stars where it is seen in emission $L\left(\mathrm{Br}_{\gamma}\right) \gtrsim 0.1-0.2 L_{\odot}$. The validity of Eq. (1) for these very luminous objects is very doubtful, and we will not consider them further in the following of this paper.

After subtraction of the photospheric component, 24 of the 28 HAe stars have circumstellar $\mathrm{Br}_{\gamma}$ emission; for these, we compute $L\left(\mathrm{Br}_{\gamma}\right)$ from $W\left(\mathrm{Br}_{\gamma}\right)_{\text {circ }}$, the continuum flux derived from the extinction-corrected $\mathrm{K}$ magnitude and the distance of the star. $L_{\text {acc }}$ is then obtained from Eq. (1). For the remaining 4, where no circumstellar emission is detected, we estimate that $W\left(\mathrm{Br}_{\gamma}\right)_{\text {circ }} \lesssim 2 \AA$, and compute upper limits to $L_{\text {acc }}$ accordingly. For HD 98922 and HD 144432, $L_{\mathrm{acc}}$ refers to the quoted distance lower limit, and is therefore also a lower limit to the true value. The results are given in Table 1 and shown in Fig. 1 as a function of the stellar luminosity. Note that Eq. (1) has been derived for objects with $L\left(\mathrm{Br}_{\gamma}\right) \lesssim 3 \times 10^{-3} L_{\odot} ; 2$ objects (HD 98922 and VV Ser) have larger $L\left(\mathrm{Br}_{\gamma}\right)$, and the use of Eq. (1) should be viewed with some caution.

3 This publication makes use of data products from the Two Micron All Sky Survey, which is a joint project of the University of Massachussetts and the Infrared Processing and Analysis Center/California Institute of Technology, funded by the National Aeronautics and Space Administration and the National Science Foundation. 
Table 1. Herbig Ae properties - Col. 1: star name; Col. 2: spectral type; Col. 3: $V$ magnitude; Col. 4: $K$ magnitude; Col. 5: $V$ extinction; Col. 6: distance; Col. 6: effective temperature; Col. 8: stellar luminosity; Col. 9: stellar mass; Col. 10: observed $\mathrm{Br}_{\gamma}$ equivalent width; Col. 11: equivalent width of the circumstellar $\mathrm{Br}_{\gamma}$; Col. 12: accretion luminosity; Col. 13: mass accretion rate.

\begin{tabular}{|c|c|c|c|c|c|c|c|c|c|c|c|c|}
\hline (1) & (2) & (3) & (4) & (5) & (6) & (7) & (8) & (9) & (10) & (11) & $(12)$ & (13) \\
\hline Name & ST & $\begin{array}{c}V \\
(\mathrm{mag})\end{array}$ & $\begin{array}{c}K \\
(\mathrm{mag})\end{array}$ & $\begin{array}{c}A_{\mathrm{V}} \\
(\mathrm{mag})\end{array}$ & $\begin{array}{c}D \\
(\mathrm{pc})\end{array}$ & $\begin{array}{l}T_{\text {eff }} \\
(\mathrm{K})\end{array}$ & $\begin{array}{l}L_{\star} \\
\left(L_{\odot}\right)\end{array}$ & $\begin{array}{c}M_{\star} \\
\left(M_{\odot}\right)\end{array}$ & $\begin{array}{c}\mathrm{Br}_{\gamma}(\mathrm{obs}) \\
(\AA)\end{array}$ & $\begin{array}{c}\mathrm{Br}_{\gamma}(\operatorname{circ}) \\
(\AA)\end{array}$ & $\begin{array}{c}\log L_{\text {acc }} \\
\quad\left(L_{\odot}\right)\end{array}$ & $\begin{array}{c}\log \dot{M}_{\mathrm{acc}} \\
\left(M_{\odot} / \mathrm{yr}\right)\end{array}$ \\
\hline HD 149914 & B9 & 6.75 & 5.69 & 0.9 & 165 & 10600 & 158 & 3.5 & $6.9 \pm 0.2$ & -0.8 & -0.51 & -7.99 \\
\hline HD 179218 & B9 & 7.2 & 5.99 & 1.8 & 240 & 10600 & 500 & 4 & $1.3 \pm 0.2$ & -10.1 & 0.70 & -6.59 \\
\hline HD 176386 & B9 & 7.30 & 6.69 & 0.5 & 140 & 10600 & 49 & 2.7 & $6.2 \pm 0.2$ & -3.1 & -0.49 & -8.11 \\
\hline HD 97300 & B9 & 9.00 & 7.15 & 1.2 & 190 & 10600 & 35 & 2.5 & $9.1 \pm 0.2$ & - & $<-0.5$ & $<-8.18$ \\
\hline HD 98922 & B9 & 6.77 & 4.28 & 0.3 & $>540$ & 10600 & $>890$ & 5: & $-2.4 \pm 0.2$ & -3.7 & 1.50 & -5.76 \\
\hline VV Ser & B9 & 11.92 & 6.32 & 3.1 & 260 & 10600 & 85 & 3.1 & $-9.0 \pm 0.2$ & -13.0 & 1.20 & -6.34 \\
\hline $51 \mathrm{Oph}$ & B9.5 & 4.81 & 4.29 & 0.1 & 131 & 10200 & 245 & 4 & $1.3 \pm 0.3$ & -5.6 & 0.54 & -6.87 \\
\hline HD 141569 & B9.5 & 7.00 & 6.82 & 0. & 99 & 10200 & 22 & 2.3 & $6.0 \pm 0.1$ & -4.5 & -0.68 & -8.37 \\
\hline NX Pup & A0 & 9.96 & 6.08 & 1.4 & 450 & 9840 & 42 & 2.5 & $-1.9 \pm 0.2$ & -2.4 & 0.58 & -6.97 \\
\hline HR 4796 & A0 & 5.78 & 5.77 & 0.1 & 67 & 9840 & 26 & 2.4 & $9.4 \pm 0.2$ & - & $<-0.9$ & $<-8.53$ \\
\hline HD 97048 & A0 & 8.46 & 5.94 & 1.0 & 180 & 9840 & 41 & 2.5 & $-6.3 \pm 0.15$ & -8.4 & 0.39 & -7.17 \\
\hline HD 104237 & A0 & 6.59 & 4.58 & 0.7 & 116 & 9840 & 6 & 2.0 & $-4.7 \pm 0.2$ & -6.7 & 0.43 & -7.45 \\
\hline HD 95881 & A1 & 8.25 & 5.73 & 0.3 & 120 & 9450 & 10 & 2 & $-1.6 \pm 0.2$ & -2.8 & -0.31 & -8.04 \\
\hline HD 150193 & A1 & 8.88 & 5.48 & 1.5 & 150 & 9450 & 30 & 2.3 & $-3.5 \pm 0.15$ & -5.5 & 0.27 & -7.29 \\
\hline HD 163296 & A1 & 6.87 & 4.78 & 0.3 & 122 & 9450 & 36 & 2.3 & $-4.7 \pm 0.1$ & -6.9 & 0.40 & -7.12 \\
\hline HD 144667 & A1.5 & 6.63 & 6.67 & 0.1 & 140 & 9300 & 51 & 2.5 & $5.8 \pm 0.15$ & -7.2 & -0.17 & -7.63 \\
\hline TY Cra & A2 & 9.42 & 6.67 & 1.3 & 130 & 9120 & 8 & 1.8 & $4.7 \pm 0.2$ & - & $<-0.6$ & $<-8.31$ \\
\hline WW Vul & A3 & 10.51 & 7.28 & 1.0 & 550 & 8670 & 30 & 2.4 & $-3.6 \pm 0.2$ & -5.4 & 0.60 & -6.89 \\
\hline HD 169142 & A5 & 8.15 & 6.41 & 0.3 & 145 & 8260 & 15 & 1.8 & $-5.0 \pm 0.1$ & -9.7 & 0.08 & -7.40 \\
\hline KK Oph & A6 & 10.57 & 5.79 & 0.44 & 160 & 8100 & 6.2 & 1.6 & $-1.2 \pm 0.15$ & -1.5 & -0.30 & -7.91 \\
\hline HD 139614 & A7 & 8.25 & 6.75 & 0.1 & 150 & 7900 & 11 & 1.8 & $2.2 \pm 0.2$ & -3.0 & -0.48 & -7.99 \\
\hline HD 142666 & A8 & 8.81 & 6.08 & 0.8 & 116 & 7580 & 8 & 1.6 & $1.0 \pm 0.2$ & -2.4 & -0.50 & -8.00 \\
\hline HD 100453 & A9 & 7.79 & 5.6 & 0.0 & 111 & 7300 & 9 & 1.7 & $1.6 \pm 0.2$ & -1.5 & -0.58 & -8.04 \\
\hline HD 144432 & A9 & 8.16 & 5.89 & 0.2 & $>200$ & 7300 & $>23$ & $>2$ & $-2.1 \pm 0.2$ & -5.1 & 0.26 & -7.07 \\
\hline HD 135344 & F3 & 8.61 & 5.84 & 0.3 & 84 & 6590 & 8 & 1.5 & $0.3 \pm 0.2$ & -1.3 & -0.93 & -8.27 \\
\hline T CrA & F5: & 12.04 & 6.60 & 1.9 & 130 & 6400 & 1.4 & 1.2 & $9.6 \pm 0.2$ & - & $<-0.6$ & $<-8.20$ \\
\hline $\mathrm{R} \mathrm{CrA}$ & F5: & 11.50 & 2.86 & 1.4 & 130 & 6400 & 1.3 & 1.2 & $-1.2 \pm 0.3$ & -1.2 & 0.50 & -7.12 \\
\hline HD 142527 & F6 & 8.33 & 4.98 & 0.7 & 200 & 6270 & 69 & 3.5 & $0.3 \pm 0.2$ & -1.2 & 0.04 & -7.16 \\
\hline AB Aur & A0 & 7.05 & 4.2 & 0.5 & 140 & 9840 & 48 & 2.4 & $-4.4 \pm 0.15$ & -5.5 & 0.63 & -6.85 \\
\hline HD 34282 & A0 & 9.85 & 7.68 & 0.6 & 400 & 9840 & 30 & 2.2 & $2.7 \pm 0.17$ & - & $<-0.20$ & $<-7.71$ \\
\hline RR Tau & A0 & 11.0 & 7.39 & 1.1 & 800 & 9840 & 37 & 2.5 & $-2.56 \pm 0.05$ & -3.5 & 0.70 & -6.86 \\
\hline HD 35187 & A2 & 7.78 & 5.91 & 0.65 & 150 & 9120 & 27 & 2.3 & $7.13 \pm 0.27$ & - & $<-0.3$ & $<-7.81$ \\
\hline UX Ori & A3 & 10.0 & 7.21 & 0.3 & 450 & 8670 & 39 & 2.3 & $-2.4 \pm 0.02$ & -3.9 & 0.32 & -7.19 \\
\hline HK Ori & A5 & 11.66 & 7.34 & 1.2 & 450 & 8260 & 12 & 2.0 & $-1.44 \pm 0.08$ & -2.2 & 0.08 & -7.50 \\
\hline HR 5999 & A6 & 7.04 & 4.39 & 0.5 & 210 & 8100 & 85 & 3.2 & $-1.74 \pm 0.01$ & -6.5 & 0.95 & -6.37 \\
\hline CQ Tau & $\mathrm{F} 2$ & 9.42 & 6.17 & 1.9 & 130 & & 6.6 & 1.5 & $3.17 \pm 0.18$ & - & $<-0.6$ & $<-8.08$ \\
\hline
\end{tabular}

Note: The last 9 lines are objects from Rodgers (2001).

The mass accretion rate follows from the relation $\dot{M}_{\text {acc }}=$ $L_{\text {acc }} R_{\star} /\left(G M_{\star}\right)$, and is shown in Table 1, Col. (13).

The uncertainties on $L_{\text {acc }}$ and $\dot{M}_{\text {acc }}$ are dominated in most cases by the uncertainties deriving from the subtraction of the veiled photospheric absorption component from the observed spectrum. The error bars shown in Fig. 1 correspond to an uncertainty on the resulting $W\left(\mathrm{Br}_{\gamma}\right)_{\text {circ }}$ of $\sim \pm 2 \AA$, which we consider realistic in the majority of cases.

For any given object, variability of lines and continuum can also be large. There are no systematic studies of time variability of the $\mathrm{Br}_{\gamma}$ equivalent width in our sample stars, but one can compare our measurements with the Rodgers (2001) results from lower resolution spectra $(R \sim 500-2000)$ for the 7 stars we have in common. The differences are within the errors for 3 of them, but larger than $2 \AA$ in 2 , one being $\mathrm{R} \mathrm{CrA}$. This confirms the well known fact that the accretion rate in pre-main sequence stars varies and that one can only estimate the "typical" value of any individual object if the results of long term time monitoring are available. However, if one is interested in the properties of a large sample of stars, as in this work, one can safely assume that their time variability will increase the spread of the determinations, but not bias the average values.

\section{Discussion}

Figure 1 plots $L_{\text {acc }}$ as a function of $L_{\star}$ for the HAe observed in this paper. We have added six HAe stars (listed at the bottom of Table 1) taken from the Rodgers (2001) thesis, for a total of 36 objects. Typically, the HAe stars have $L_{\text {acc }}$ roughly between $10 \%$ and $1 \% L_{\star}$. There are very few objects with $L_{\text {acc }}>0.1 L_{\star}$, contrary to what happens for lower mass objects in Taurus and Ophiuchus (see Natta et al. 2006; Calvet et al. 2004).

Figure 2, top Panel, shows the $\dot{M}_{\text {acc }}$ distribution of the HAe stars; the values of $\dot{M}_{\text {acc }}$ cover the interval $\sim 10^{-6}-\lesssim 3 \times$ $10^{-9} M_{\odot} / \mathrm{yr}$, but few stars $(25 \%)$ have $\dot{M}_{\text {acc }}>10^{-7} M_{\odot} / \mathrm{yr}$; $36 \%$ have $\dot{M}_{\text {acc }}<10^{-8} M_{\odot} / \mathrm{yr}$. The median value is $\dot{M}_{\mathrm{acc}}=$ $3.5 \times 10^{-8} M_{\odot} / y r$. These results agree with those obtained by Muzerolle et al. (2004), who found that intermediate-mass objects have typical $\mathrm{H}_{\alpha}$ profiles and/or Balmer discontinuity roughly consistent with the predictions of magnetospheric 


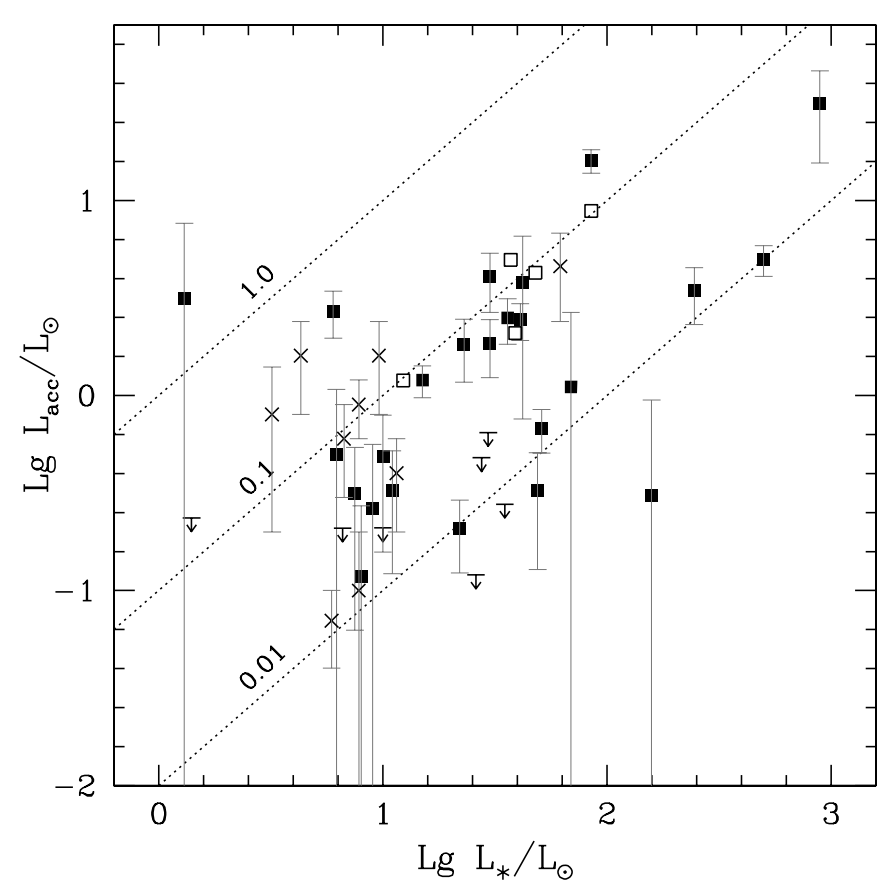

Fig. 1. Accretion luminosity as a function of $L_{\star}$ for our sample (filled squares) and the Rodgers (2001) objects (open squares); arrows show upper limits. For comparison, we show also the IMTTS $L_{\text {acc }}$ measured by Calvet et al. (2004) from veiling (crosses).

accretion models with accretion rates $\lesssim 10^{-7} M_{\odot} /$ yr. They studied in detail only one object, UX Ori, for which they found a value $\dot{M}_{\text {acc }} \sim 10^{-8} M_{\odot} / \mathrm{yr}$, comparable with the value $\left(\sim 6 \times 10^{-8} M_{\odot} / \mathrm{yr}\right)$ we derive from the low resolution spectrum of Rodgers (2001).

Our results support the conclusion of Muzerolle et al. (2004) that in most HAe stars the accretion rate is sufficiently low that the gas in the inner disk, inside the dust evaporation radius, is optically thin, allowing the direct irradiation of the inner dust edge (the rim) by the star. One of the conditions for the formation of a puffed-up rim (Natta et al. 2001) seems therefore generally verified.

Figure 3 plots $\dot{M}_{\text {acc }}$ as a function of $M_{\star}$ for the HAe stars, together with objects in Ophiuchus (Natta et al. 2006), Taurus (Muzerolle et al. 2005, and references therein; Mohanty et al. 2005), and IMTTS in Orion and Taurus (Calvet et al. 2004). Figure 2 compares the $\dot{M}_{\text {acc }}$ distribution of the HAe stars to that of objects in Ophiuchus in two mass ranges, as labelled. One can see that the median $\dot{M}_{\text {acc }}$ for the HAe stars $\left(\sim 3 \times 10^{-8} M_{\odot} / \mathrm{yr}\right)$ is about a factor ten higher than for Ophiuchus stars with $0.3<$ $M_{\star}<1 M_{\odot}$, and a factor 100 larger than in the very low mass objects with $M_{\star}<0.3 M_{\odot}$. This is roughly consistent with the predictions of the $\dot{M}_{\text {acc }} \propto M_{\star}{ }^{1.8}$ relation derived for subsolar mass objects. A close inspection of Fig. 3, however, shows that the HAe stars lack very strong accretors, as already noted in discussing Fig. 1. If the upper envelope of the distributions observed in Taurus and Ophiuchus extended to HAe stars, we would expect a much larger fraction of objects accreting at rates higher than $10^{-7} M_{\odot} / y r$.

It is not easy, at this stage, to understand why this happens. The HAe stars form a sample which is not complete nor homogeneous. The objects are located in a variety of star forming regions, and, in some cases, are isolated in the sky. We think that the most likely interpretation of their behaviour is "aging", since the accretion rate in viscous disks with constant $\alpha$ parameter

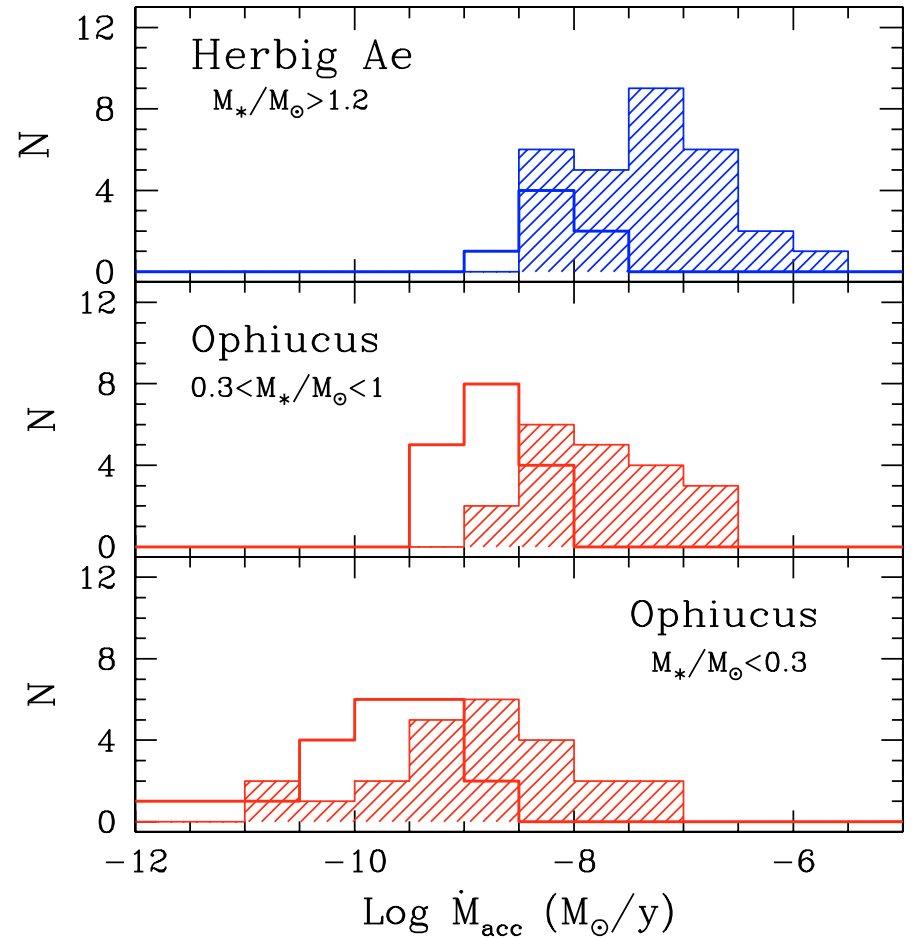

Fig. 2. Distribution of the mass accretion rate in HAe stars (top panel). The shadowed boxes show actual measurements, the empty boxes (thick histogram) the distribution of the upper limits. The mid and bottom panels show the distribution of $\dot{M}_{\text {acc }}$ for Class II objects in Ophiuchus with mass $0.3<M_{*} / M_{\odot}<1$ and $M_{*} / M_{\odot} \leq 0.3$, respectively (data from Natta et al. 2006).

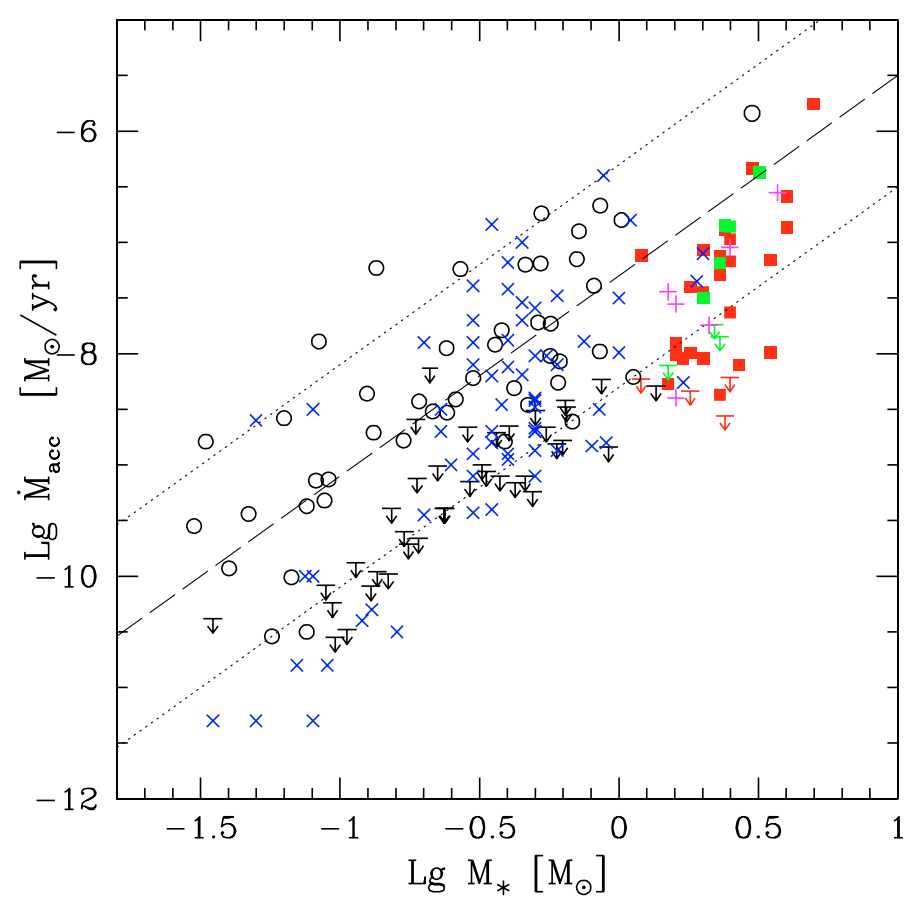

Fig. 3. Mass accretion rate as a function of $M_{\star}$ for HAe stars (filled squares and upper limits), Ophiuchus Class II objects (open dots and upper limits; Natta et al. 2006), Taurus CTTS and BDs (crosses; Muzerolle et al. 2005; Mohanty et al. 2005; Calvet et al. 2004; and references therein), IMTTS in Orion (plus signs, Calvet et al. 2004). The dashed line shows the best-fit to the Ophiuchus data $\left(\dot{M}_{\text {acc }} \propto M_{\star}{ }^{1.8}\right)$; the dotted lines are \pm 1 dex. 
Table 2. Herbig Be properties - Col. 1: star name; Col. 2: spectral type; Col. 3: observed $\mathrm{Br}_{\gamma}$ equivalent width; Col. 4: $\mathrm{Br}_{\gamma}$ luminosity.

\begin{tabular}{lccc}
\hline \hline$(1)$ & $(2)$ & $(3)$ & $(4)$ \\
Name & $\mathrm{ST}$ & $\mathrm{Br}_{\gamma}(\mathrm{obs})$ & $\log L\left(\mathrm{Br}_{\gamma}\right)$ \\
& & $(\AA)$ & $\left(L_{\odot}\right)$ \\
\hline V921 Sco & B0 & $-17.3 \pm 0.25$ & $>-0.7$ \\
MWC 300 & B0-B1 & $-10.9 \pm 0.25$ & $>-0.8$ \\
MWC 166 & B0 IV & $2.5 \pm 0.2$ & - \\
MWC 297 & B1.5 & $-16.0 \pm 0.2$ & $>-0.6$ \\
\hline
\end{tabular}

Table 3. $\mathrm{Br}_{\gamma}$ equivalent width of template stars - Col. 1: spectral type; Col. 2: adopted $\mathrm{Br}_{\gamma}$ equivalent widht.

\begin{tabular}{cc}
\hline \hline$(1)$ & $(2)$ \\
ST & $W\left(\mathrm{Br}_{\gamma}\right)$ \\
& $(\AA)$ \\
\hline B8 & 11.4 \\
A0 & 9.7 \\
A2 & 13.4 \\
A8 & 12.0 \\
F3 & 6.6 \\
F6 & 5.5 \\
\hline
\end{tabular}

is expected to deacrease with time roughly as $t^{-1.5}$ (Hartmann et al. 1998). HAe stars are, on average, older than Ophiuchus objects, which cluster at ages $~ 0.5-1$ Myr (Palla \& Stahler 2000). Their nominal ages, obtained by comparing their location on the HR diagram with Palla \& Stahler (1999) evolutionary tracks, range from $\sim 1$ to $>10 \mathrm{Myr}$; several stars are close to the ZAMS, and it is in fact interesting that 6/7 stars with non-detected $\mathrm{Br}_{\gamma}$ emission are ZAMS objects, the exception being HD 35187, with an estimated age $\sim 8$ Myr. However, one should not overinterpret these findings, as ages of intermediate-mass stars derived from their location on the HR diagram are very uncertain.

It is possible that the steep dependence of $\dot{M}_{\text {acc }}$ on $M_{\star}$ does not extend to objects more massive than, say, $\sim 1 M_{\odot}$, reflecting a change in the physical processes that control disk evolution and/or formation, and which have been discussed in a number of recent papers (e.g., Muzerolle et al. 2003; Natta et al. 2006; Padoan et al. 2004; Alexander \& Armitage 2006; Dullemond et al. 2006). Unfortunately, given the lack of completeness and the selection biases that affect it, it is difficult to establish the relevance of the HAe sample for this ungoing discussion. It is worth to remind, in this context, the well known bias due to the spectral type selection, which introduces an age-mass correlation in the sample (e.g., van Boekel et al. 2005) and automatically excludes young, relatively low mass $\left(\lesssim 2.5 M_{\odot}\right)$ objects.

\section{Conclusions}

We have reported in this paper measurements of the disk mass accretion rates in a sample of 36 Herbig Ae stars. The values of $\dot{M}_{\text {acc }}$ are derived from the measured luminosity of the $\mathrm{Br}_{\gamma}$ emission line, using the correlation between $L\left(\mathrm{Br}_{\gamma}\right)$ and the accretion luminosity $L_{\text {acc }}$, established by Muzerolle et al. (1998) and Calvet et al. (2004). Note that it is an empirical correlation, which makes no assumptions on the origin of $\mathrm{Br}_{\gamma}$.

We find that $80 \%$ of the stars, all of which have evidence of an associated circumstellar disk, are accreting matter, with rates $3 \times 10^{-9} \lesssim \dot{M}_{\text {acc }} \lesssim 10^{-6} M_{\odot} /$ yr; for 7 objects, 6 of which are located on the ZAMS in the HR diagram, we do not detect any line emission. Few HAe stars $(25 \%)$ have $\dot{M}_{\text {acc }}>10^{-7} M_{\odot} /$ yr. These results support the conclusion of Muzerolle et al. (2004) that in most HAe stars the accretion rate is sufficiently low that the gas in the inner disk, inside the dust evaporation radius, does not prevent the formation of the puffed-up rim, proposed by Natta et al. (2001) and Dullemond et al. (2001).

When compared to the $\dot{M}_{\text {acc }}$ values found for lower-mass stars in the star forming regions Taurus and Ophiuchus, HAe stars have on average higher accretion rates; however, there is a lack of very strong accretors among them, as shown by the very few objects with $L_{\text {acc }}>10 \% L_{\star}$. It seems likely that this is because on average HAe stars are older than TTS in Ophiuchus or Taurus. However, ages of intermediate-mass stars derived from the HR diagram location are very uncertain; this, and the various selection biases which plague the HAe sample, make the comparison very difficult.

When we consider individual stars, the largest uncertainties on $\dot{M}_{\text {acc }}$ come from line variability, as in TTS, but also from the presence of a photospheric absorption component of $\mathrm{Br}_{\gamma}$ in early-type stars, which needs to be subtracted from the observed one, and from the $K$-band excess emission. A better characterization of the photospheric profiles of template stars would be very valuable.

Acknowledgements. We thank the ESO staff for competent and efficient support during the preparation of the observing blocks and for the excellent data delivered for this service observing programme. The spectroscopic observations of template stars were kindly provided by Bernadette Rodgers. This publication makes use of data products from the Two Micron All Sky Survey, which is a joint project of the University of Massachusetts and the Infrared Processing and Analysis Center/California Institute of Technology, funded by the National Aeronautics and Space Administration and the National Science Foundation. This project was partially supported by MIUR grant 2004025227/2004.

\section{Appendix $\mathbf{A}: \mathrm{Br}_{\gamma}$ spectra}

The observed spectra, normalized to the continuum level and shifted for an easier display, are shown in Fig. A.1. They are ordered roughly according to the observed $W\left(\mathrm{Br}_{\gamma}\right)_{\text {obs }}$, from strong emission to strong absorption. In HAe, the strongest peak emission (HD 97048) is about $44 \%$ of the continuum; the deepest absorption (HR 4796) is $15 \%$ of the continuum.

The resolution of the spectra $\left(\sim 30 \mathrm{~km} \mathrm{~s}^{-1}\right)$ allows us to resolve the lines in all objects. The observed line shape results from the superposition of the circumstellar emission, the photospheric absorption and the veiling $K$-band continuum, very likely from the disk itself. One can see the signature of the broad photospheric absorption component in several objects, where the $K$-band veiling is moderate or absent (e.g., HD 149914 , HD 97300, HR 4796). In some objects both the circumstellar emission and the photospheric absorption are clearly visible (e.g., HD 141569 and $51 \mathrm{Oph}$ ); in both objects the emission has a double-peaked profile with peak-to-peak separation $\sim 300 \mathrm{~km} \mathrm{~s}^{-1}$.

In general, the emission lines are very broad, with half width at $10 \%$ of peak intensity $(10 \% \mathrm{HW})$ of $200-300 \mathrm{~km} \mathrm{~s}^{-1}$. VV Ser has very broad wings, which extends to $\pm 450 \mathrm{~km} \mathrm{~s}^{-1}$ from the line center. As a reference, the escape velocity of a typical A0 star $\left(M_{\star}=2.3 M_{\odot}, R=4 R_{\odot}\right)$ is $330 \mathrm{~km} \mathrm{~s}^{-1}$; the $10 \% \mathrm{HW}$ of $\mathrm{Br}_{\gamma}$ is often close to this value.

In comparison, the three early B objects with emission have very strong lines. They are relatively narrow $(10 \% \mathrm{HW} \lesssim$ $120 \mathrm{~km} \mathrm{~s}^{-1}$ ) when compared to the estimated escape velocity, which are of $\sim 200 \mathrm{~km} \mathrm{~s}^{-1}$.

Clearly, the study of the profiles of the near-IR hydrogen emission lines in these intermediate-mass objects promises very 


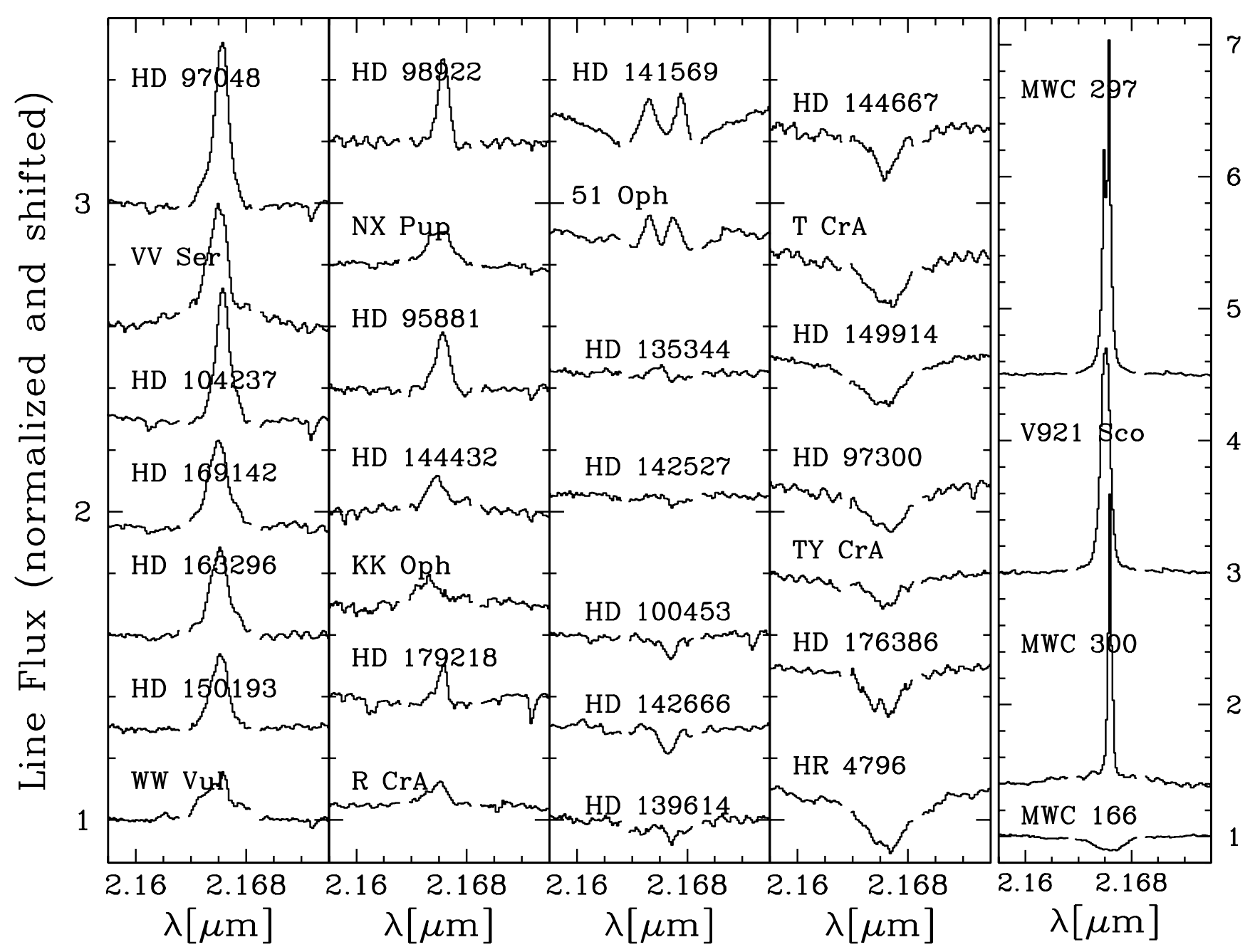

Fig. A.1. Observed $\mathrm{Br}_{\gamma}$ profiles. The observed fluxes have been normalized to the continuum and shifted for easier display. The stars are roughly order according to the observed $W\left(\mathrm{Br}_{\gamma}\right)_{\mathrm{obs}}$, from strong emission (left-top) to strong absorption (right-bottom). The strongest peak emission (HD 97048) is about 44\% of the continuum; the deepest absorption (HR 4796) is 15\% of the continuum. The four early B stars are displayed in the fifth panel to the right, with a different vertical scale.

interesting results, and should be pursued further, possibly with even higher resolution spectrometers.

\section{References}

Alexander, R. D., \& Armitage, Ph.J. 2006, ApJ 639, L83 Calvet, N., Muzerolle J., Briceño C., et al. 2004, ApJ, 128, 1294 Clarke, C. J., \& Pringle, J. E. 2006, MNRAS, 370, L10 Dullemond, C., Dominik C., \& Natta, A. 2001, ApJ, 560, 957 Dullemond, C., Natta, A., \& Testi, L. 2006, A\&A, 645, L69 Gullbring, E., Hartmann, L., Briceño, C., \& Calvet, N. 1998, ApJ, 492, 323 Hartmann, L., Calvet, N., Gullbring, E., \& D’Alessio P., 1998, ApJ, 495, 385 Hernández, J., Calvet, N., Hartmann, L., et al. 2005, AJ, 129, 856 Isella, A., \& Natta, A. 2005, A\&A, 438, 899

Malbet, F., Benisty M., De Wit, W. J., et al. 2006, A\&A, accepted [arXiv: astro-ph/0510350]

Meeus, G., Waters, L. B. F. M., Bouwman J., et al. 2001, A\&A, 356, 476 Mohanty, S., Jayawardhana, R., \& Basri, G. 2005, ApJ, 626, 498 Muzerolle, J., Hartmann, L., \& Calvet, N. 1998, AJ, 116, 2965

Muzerolle, J., Hillenbrand, L., Calvet, N., Briceño, C., \& Hartmann, L. 2003, ApJ, 592, 266
Muzerolle, J., D’Alessio, P., Calvet, N., \& Hartmann, L. 2004, ApJ, 617, 406 Muzerolle, J., Luhman, K. L., Briceño C., Hartmann, L., \& Calvet, N. 2005, ApJ, 625,906

Natta, A., Prusti T., Neri R., Grinin V. P., \& Mannings, V. 2001, A\&A, 371, 186 Natta, A., Testi, L., Muzerolle, J., et al. 2004, A\&A, 424, 603

Natta, A., Testi, L., \& Randich, S. 2006, A\&A, 452, 245

Padoan P., Kritsuk A., Norman, M. L., \& Nordlund, Å. 2004, ApJ, 622, L61

Palla, F., \& Stahler, S. W. 1993, ApJ, 418, 414

Palla, F., \& Stahler, S. W. 1999, ApJ, 525, 772

Palla, F., \& Stahler, S. W. 2000, ApJ, 540, 255

Rodgers B. 2001, Ph.D. Thesis, University of Washington

Tatulli, E., Isella A., Natta A., et al. 2006, A\&A, submitted

Testi, L., Palla F., \& Natta, A. 1998, A\&AS, 133, 81

van den Ancker, M. E., The P. S., Tjin A Djie, H. R. E., et al. 1997, A\&A, 324, L33

van den Ancker, M. E., de Winter, D., \& Tjin A Djie, H. R. E. 1998, A\&A, 330, 145

van den Ancker, M. 2004, in High Resolution Infrared Spectroscopy in Astronomy, ed. H.-U. Käufl, R. Siebenmorgen, \& A. Moorwood van Boekel, R., Min M., Waters, L. B. F. M., et al. A\&A, 437, 189

Wallace, L., \& Hinkle, K. 1997, ApJS, 111, 445

White, R. J., \& Ghez, A. 2001, ApJ, 556, 265 